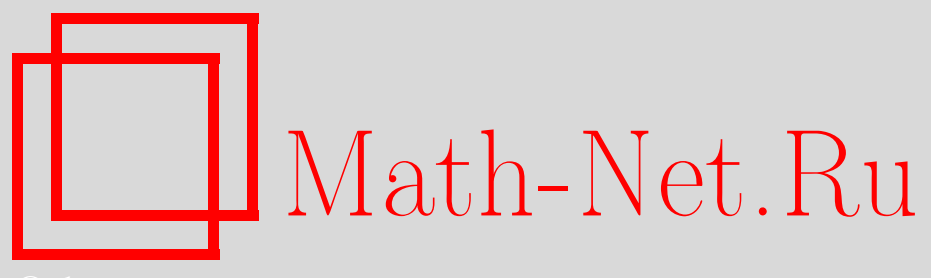

В. М. Бабич, В. С. Буслаев, А. М. Вершик, С. Г. Гиндикин, С. В. Кисляков, А. А. Лаптев, В. А. Марченко, Н. К. Никольский, Л. А. Пастур, Б. А. Пламеневский, М. З. Соломяк, Т. А. Суслина, Н.Н.Уральцева, Л. Д. Фаддеев, В. П. Хавин, Д. Р. Яфаев, Михаил Шлемович Бирман (некролог), УМН, 2010, том 65, выпуск $3,185-190$

DOI: https://doi.org/10.4213/rm9352

Использование Общероссийского математического портала Math-Net.Ru подразумевает, что вы прочитали и согласны с пользовательским соглашением http://www . mathnet.ru/rus/agreement

Параметры загрузки:

IP : 54.197 .217 .227

26 апреля 2023 г., $14: 23: 36$

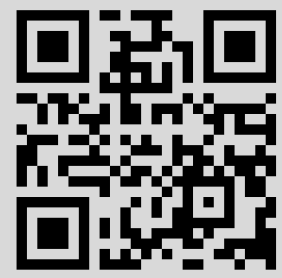




\section{Михаил Шлемович Бирман}

2 июля 2009 г. ушел из жизни Михаил Шлемович Бирман - выдающийся математик, один из мировых лидеров в спектральной теории операторов.

Михаил Шлемович Бирман родился 17 января 1928 г. в Ленинграде. Его отец был ученым специалистом по теоретической механике, профессором. Мать была школьной учительницей.

М.Ш. Бирман учился на математико-механическом факультете Ленинградского государственного университета в 1946-1950 гг. Специализировался по методам вычислений. Своими учителями Михаил Шлемович считал Марка Константиновича Гавурина, который был руководителем его дипломной работы, и Леонида Витальевича Канторовича. Еще будучи студентом, М.Ш. Бирман подрабатывал в Ленинградском отделении Математического института в лаборатории Канторовича. Он был лучшим студентом на курсе, с высоким интеллектом и независимым

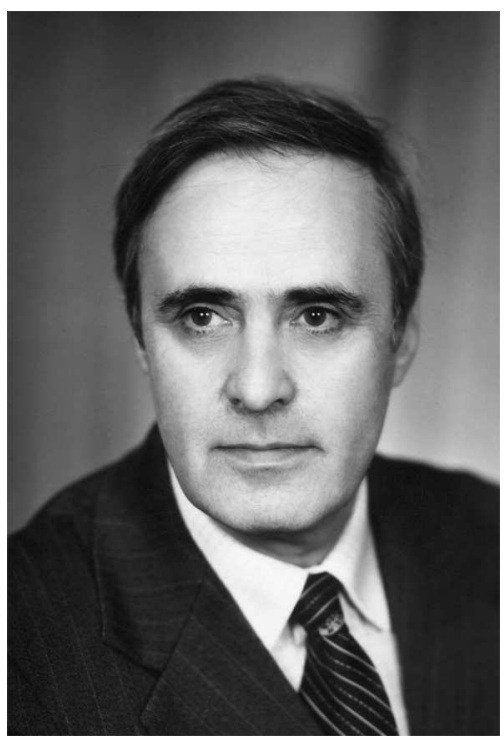
мышлением. Но в аспирантуру его не взяли из-за тогдашней политики антисемитизма.

В 1947 г. Михаил Шлемович женился на своей однокурснице Татьяне Петровне Ильиной. В 1948 г. у них родился сын Женя. С Татьяной Петровной они счастливо прожили всю жизнь. Она ушла из жизни двумя годами раньше него. Она всегда была его добрым ангелом-хранителем. Благодаря ее заботе и любви он мог полностью посвятить себя своему любимому делу - занятиям математикой.

После окончания университета М.Ш. работал в Ленинградском горном институте на кафедре высшей математики ассистентом. В 1954 г. успешно защитил кандидатскую диссертацию.

Большую роль в научном становлении Михаила Шлемовича сыграло его активное участие в работе ленинградского семинара по математической физике, организованного в начале 50-х годов по инициативе Владимира Ивановича Смирнова. Впоследствии в течение многих лет Михаил Шлемович вместе с Ольгой Александровной Ладыженской руководил этим семинаром.

По предложению Владимира Ивановича и Ольги Александровны, в 1956 г. Михаил Шлемович перешел работать в ЛГУ, на кафедру математической физики физического факультета. Там он проработал до конца своей жизни - более 50 лет. В 1962 г. он защитил докторскую диссертацию на тему "Спектр сингулярных граничных задач". М.Ш. Бирман был блестящим лектором. Он был одним из лидеров кафедры, и не только научным. Он не только обладал высочайшим профессиональным авторитетом, но всегда был очень внимателен к людям. Многие его младшие коллеги 
испытывали его влияние как прекрасного профессионала и мудрого человека. У него всегда были очень высокие профессиональные стандарты - как к качеству результатов, так и к изложению материала. И прежде всего эти требования он предъявлял самому себе.

Михаил Шлемович вместе со своим соратником Михаилом Захаровичем Соломяком создал сильную научную школу по спектральной теории операторов, известную во всем мире. Многие его ученики сами стали известными учеными и работают сейчас в ведущих университетах России и на Западе.

Михаил Шлемович Бирман - автор более 160 научных статей и двух книг. Он много занимался редакторской деятельностью, был членом редколлегий журналов РАН "Алгебра и анализ", "Функциональный анализ и его приложения". Заслуги М.Ш. Бирмана были отмечены почетными званиями "Заслуженный деятель науки РФ", "Почетный профессор СПбГУ". В 2008 г. он получил награду Правительства Санкт-Петербурга и Санкт-Петербургского научного центра РАН - премию имени П. Л. Чебышёва в области математики и механики.

Работы М.Ш. Бирмана получили международное признание и обильно цитируются в математической литературе. Он многократно был пленарным докладчиком на международных конференциях. Его приглашали с научными визитами в ведущие университеты и научные центры мира.

Остановимся кратко на главных научных достижениях М. Ш. Бирмана.

Его первые работы (1950-1952) выполнены под влиянием Канторовича и Гавурина. Они посвящены вычислительным вопросам в задачах линейной алгебры, а именно многошаговому итерационному методу наискорейшего спуска. Разработанные им методы были внедрены в практику расчетов.

Следующий важный цикл работ Михаила Шлемовича (1953-1962) относится к теории расширений симметричных положительно определенных операторов и применению этой теории к эллиптическим граничным задачам. Он пришел к этой тематике, занимаясь вычислительными методами, связанными с вариационным подходом к решению граничных задач. Осознав роль общей теории расширений в этом круге вопросов, он стал изучать работы Марка Григорьевича Крейна и Марка Иосифовича Вишика по этой теории. Особенно большое впечатление на него произвели работы Крейна, которые, по словам М.Ш., открыли для него новый мир. С тех пор Михаил Шлемович всегда считал себя "заочным учеником" М. Г. Крейна. Развивая идеи Крейна и Вишика, М.Ш. Бирман построил параметрическое описание всех самосопряженных расширений данного симметричного положительно определенного оператора. Этот подход был успешно применен в работах по вариационным методам.

Вместе с тем, М.Ш. Бирман обнаружил и другие, еще более важные приложения своих результатов по теории расширений. Речь идет об устойчивости существенного спектра для эллиптических операторов в неограниченных областях. Именно, он установил, что существенный спектр устойчив относительно изменения компактной части границы или граничных условий на такой части границы. Были получены оценки собственных значений для разности резольвент одного и того же эллиптического оператора с различными граничными условиями.

В знаменитой работе М.Ш. Бирмана "О спектре сингулярных граничных задач" (Матем. сб., 1961; краткое изложение - в заметке 1959 г., Доклады АН СССР) изучалась устойчивость существенного спектра при изменении коэффициентов оператора. Типичный пример - сравнение спектров оператора $H_{0}=-\Delta$ и оператора Шрёдингера $H=-\Delta+V$, действующих в $L_{2}\left(\mathbb{R}^{d}\right)$. Здесь $V(\mathbf{x}) \rightarrow 0$ при $|\mathbf{x}| \rightarrow \infty$. Результаты этой работы дают новый взгляд и на общую теорию возмущений. Основной прием, который можно назвать "фирменным знаком" М.Ш. Бирмана, состоит в последовательном применении квадратичных форм. Явного описания “действия" оператора, 
а также его области определения при этом удается избежать. В частности, М. Ш. Бирман широко использует понятие, позднее названное "относительной компактностью в смысле квадратичных форм".

М.Ш. Бирман показал, что для полуограниченного снизу самосопряженного оператора $A$ существенный спектр устойчив при возмущениях относительно компактным оператором $V$ (в смысле квадратичных форм). Пусть $V \geqslant 0$ и рассматривается оператор $A+\alpha V, \alpha<0$. Тогда спектр оператора $A+\alpha V$, лежащий левее спектра $A$, дискретен. Михаил Шлемович нашел соотношение, связывающее функцию распределения дискретного спектра оператора $A+\alpha V$ с функцией распределения собственных значений компактного оператора, порожденного формой оператора $V$ в энергетическом пространстве оператора $A$. Это соотношение двумя годами позже было переоткрыто Швингером и носит название принципа Бирмана-Швингера.

Сейчас принцип Бирмана-Швингера - исходная точка при изучении дискретного спектра операторов с непустым существенным спектром. Особенно часто он применяется в задачах квантовой механики.

Общие результаты были применены к дифференциальным операторам. В частности, для оператора Шрёдингера $H=-\Delta-V(\mathbf{x})$ в $L_{2}\left(\mathbb{R}^{3}\right)$ была получена знаменитая оченка Бирмана-Швингера числа отрицательных собственных значений.

Особо нужно выделить работы Михаила Шлемовича по математической теории рассеяния (60-е годы), которые оказали значительное влияние на становление и развитие этой области.

В 1957 г. Т. Като и М. Розенблюм установили следующий фундаментальный факт. Пусть $A$ и $B$ - самосопряженные операторы в гильбертовом пространстве $H, P_{\text {ас }}(A)$ ортопроектор на абсолютно непрерывное подпространство $H_{\mathrm{ac}}(A)$ оператора $A$. Предположим, что $A-B \in \mathfrak{S}_{1}$, где $\mathfrak{S}_{1}-$ класс ядерных операторов. Тогда при $t \rightarrow \pm \infty$ существуют сильные пределы операторов $e^{i B t} e^{-i A t} P_{\mathrm{ac}}(A)$, называемые волновыми операторами. Волновые операторы $W_{ \pm}(B, A)$ изометричны на $H_{\mathrm{ac}}(A)$ и $B W_{ \pm}=W_{ \pm} A$. Отсюда выводится, что абсолютно непрерывные части операторов $A$ и $B$ унитарно эквивалентны. Оператор рассеяния $S=W_{+}^{*} W_{-}$коммутирует с $A$. Если представить пространство $H_{\text {ас }}(A)$ в виде прямого интеграла, в котором диагонализуется оператор $A_{\text {ac }}$, то $S$ действует как умножение на оператор-функцию $S(\lambda)$, называемую матрицей рассеяния.

Для приложений к квантовой механике особенно важно рассмотрение оператора Шрёдингера. Тогда $A=-\Delta$ и $B=-\Delta+V(\mathbf{x})$, где $V(\mathbf{x})$ - вещественная функция, причем $V(\mathbf{x})$ стремится к нулю достаточно быстро при $|\mathbf{x}| \rightarrow \infty$. Теорема Розенблюма-Като не может быть прямо применена к этому случаю, поскольку оператор умножения на $V(\mathbf{x})$ в $L_{2}\left(\mathbb{R}^{d}\right)$ не является компактным.

Вклад М.Ш. Бирмана в решение этой проблемы был решающим. Его идея состояла в том, чтобы рассмотреть подходящие функции $\varphi$ от операторов $A$ и $B$ и применить теорему Розенблюма-Като к операторам $\varphi(A), \varphi(B)$. Он показал, что при условии ядерности оператора $\varphi(A)-\varphi(B)$ не только совпадают абсолютно непрерывные спектры операторов $A$ и $B$, но и существуют волновые операторы $W_{ \pm}(B, A)$ и $W_{ \pm}(B, A)=W_{ \pm}(\varphi(B), \varphi(A))$. Этот результат, доказанный Михаилом Шлемовичем для широкого класса функций $\varphi$, был позднее назван принципом инвариантности.

Важный вклад в теорию рассеяния был сделан в работах Михаила Шлемовича, совместных с Марком Григорьевичем Крейном. Они распространили теорему Розенблюма-Като на случай унитарных операторов. Это соответствует принципу инвариантности для дробно-линейной функции $\varphi$. Теорема Бирмана-Крейна показывает, что для пары самосопряженных операторов $A, B$ волновые операторы $W_{ \pm}(B, A)$ существуют, если разность резольвент ядерная. Этот результат уже непосредственно применим к оператору Шрёдингера.

Совместно с С. Б. Энтиной, М. Ш. Бирман последовательно развил стационарную схему в ядерной теории рассеяния. В рамках этого подхода он ввел важное понятие 
локальных волновых операторов, связанных с каким-либо интервалом спектральной оси. С их помощью М.Ш. Бирман нашел очень общие условия существования "глобальных" волновых операторов. Позднее Михаил Шлемович вернулся к этой тематике, развив совместно с Д. Р. Яфаевым общую стационарную схему вне рамок конкретных предположений ядерного или гладкого типов.

$\mathrm{C}$ теорией рассеяния тесно связано исследование функции спектрального сдвига. Для пары самосопряженных операторов $A, B$ при определенных условиях имеет место формула следа $\operatorname{Tr}(\varphi(B)-\varphi(A))=\int_{-\infty}^{\infty} \varphi^{\prime}(\lambda) \xi(\lambda) d \lambda$. Функцию $\xi(\lambda)$ называют функицей спектрального сдвига (ФСС). Понятие ФСС в теории возмущений было введено в начале 50-х годов физиком И. М. Лифшицем. Вскоре после этого М. Г. Крейн развил математическую теорию ФСС. Он оправдал формулу следа для самосопряженных операторов $A, B$ с ядерной разностью и для широкого класса функций $\varphi$.

Совместно М.Ш. Бирман и М. Г. Крейн в 1962 г. установили ставшую знаменитой формулу Бирмана-Крейна - связь между ФСС и матрицей рассеяния. Они показали, что при $A-B \in \mathfrak{S}_{1}$ разность $S(\lambda)-I$ ядерна при почти всех $\lambda \in \sigma_{\mathrm{ac}}(A)$. Тогда детерминант $S(\lambda)$ корректно определен и справедлива формула $\operatorname{Det} S(\lambda)=\exp (-2 \pi i \xi(\lambda))$. Эта формула, как и упоминавшийся выше принцип Бирмана-Швингера, стали настолько знаменитыми, что обычно цитируются без упоминания конкретных статей.

Работа Бирмана и Крейна явилась основой для дальнейших исследований многими авторами (включая самого М.Ш.) свойств $\xi(\lambda)$ и $S(\lambda)$.

В 1965 г. началась совместная работа М.Ш. Бирмана с М. З. Соломяком. Первый большой и важный цикл их совместных работ (1965-1973) посвящен систематическому изучению ДОИ (двойных операторных интегралов). М.Ш. Бирман заинтересовался ДОИ потому, что в теории рассеяния возникла техническая потребность в этом аппарате. В дальнейшем выяснилось, что ДОИ имеют приложения в самых разных областях, в первую очередь - в спектральной теории возмущений. В частности, в терминах таких интегралов удается найти удобное представление разности $\varphi(B)-\varphi(A)$ через исходные операторы. Здесь $A$ и $B$ - самосопряженные операторы а $\varphi$ - некоторая функция. Это представление было оправдано М. Ш. Бирманом и М. З. Соломяком для широкого класса функций $\varphi$ и нашло многочисленные применения.

Совместно с М. З. Соломяком, М.Ш. Бирман предложил новый подход к аппроксимации функций из классов Соболева $W_{p}^{l}(\Omega)$ (работы $60-\mathrm{x}, 70-$ х годов).

Хотя непосредственным поводом для этого послужили нужды теории ДОИ, основные применения лежат далеко за ее рамками. Наиболее яркий результат, полученный на основе этого метода (в 1967 г.), - это точные по порядку оценки $\varepsilon$-энтропии вложения пространств Соболева $W_{p}^{l}$ в $C$ или в $L_{q}$ в случаях, когда такое вложение компактно. Именно, было показано, что порядок оценки зависит только от гладкости $l$, но не от показателя $p$.

Еще одно приложение - оценки и асимптотики спектра для широкого класса интегральных операторов (статья 1977 г. в УМН).

Результаты по кусочно полиномиальным приближениям оказались также чрезвычайно полезными при доказательстве оценок и асимптотических формул для дискретного спектра дифференциальных операторов. М.Ш. Бирман и М.З. Соломяк разработали новый вариант вариационного подхода, который позволил доказывать спектральные асимптотики при минимальных предположениях о данных задачи. Например, для весового уравнения $-\Delta u=\lambda V(\mathbf{x}) u$ в ограниченной области пространства $\mathbb{R}^{d}$ классическая формула Вейля для спектра задачи Дирихле была оправдана без каких-либо предположений о границе и при условии на весовую функцию $V$, близком к необходимому. Впоследствии при $d \geqslant 3$ необходимое и достаточное условие $V \in L_{d / 2}$ в этой задаче было получено Г. В. Розенблюмом. 
Вейлевская асимптотика спектра в задаче Дирихле была оправдана для произвольных эллиптических операторов второго порядка, заданных в дивергентной форме, без каких-либо предположений о гладкости коэффициентов и границы (в задаче Неймана - в случае липшицевой границы). Были найдены и асимптотические формулы для более общих задач, включая случай эллиптических систем.

Впервые была получена асимптотика спектра для ПДО отрицательного порядка, а также исследованы спектральные оценки и асимптотики для вариационных задач со связями.

У М.Ш. всегда был интерес к изучению операторов математической физики в негладких областях и с негладкими коэффициентами. В частности, он считал важным исследовать эти вопросы для оператора Максвелла. Основная трудность здесь в том, что оператор неполуограничен и поэтому нельзя использовать подход через квадратичные формы. В связи с этим свойства решений не допускают описания в терминах стандартных пространств Соболева. Другая трудность - необходимость учета условий соленоидальности.

М.Ш. Бирман атаковал различные задачи, связанные с оператором Максвелла, на протяжении многих лет. В работах, совместных с М. З. Соломяком (1987 г.), было дано исчерпывающее описание особенностей электрической компоненты электромагнитного поля в областях с липшицевой границей. Базируясь на этом результате, авторы оправдали вейлевскую асимптотику частот электромагнитных колебаний для ограниченных областей с липшицевыми границами. Окончательный результат в этом направлении получен совсем недавно - в 2007 г. М.Ш. Бирман совместно со своим учеником Н. Д. Филоновым доказал вейлевскую асимптотику спектра для оператора Максвелла в общем случае - в области с липшицевой границей при негладких коэффициентах.

Одна из задач, к исследованию которой М.Ш. Бирмана многократно возвращался, касается исследования числа отрицательных собственных значений оператора $-\Delta-\alpha V$ в $L_{2}\left(\mathbb{R}^{d}\right)$, где потенциал $V(\mathbf{x})$ стремится к нулю на бесконечности и $\alpha>0-$ константа связи. Если $V \in L_{d / 2}\left(\mathbb{R}^{d}\right)$ и $d \geqslant 3$, то из упомянутого выше результата Г. В. Розенблюма вытекает, что это число, обозначаемое $N_{-}(-\Delta-\alpha V)$, удовлетворяет асимптотическому закону Вейля. Назовем этот случай "регулярным".

Может быть и нерегулярный случай, когда потенциал убывает медленно и не принадлежит классу $L_{d / 2}\left(\mathbb{R}^{d}\right)$, но число отрицательных собственных значений оператора $-\Delta-\alpha V$ конечно. Эффекты, возникающие в нерегулярном случае, были подробно исследованы М.Ш. Бирманом совместно с М. З. Соломяком (1988-1992). Были получены соответствующие оценки и асимптотики. Выяснилось, что для любого заданного $q>d / 2$ можно указать класс потенциалов $V$, для которых $N_{-}(-\Delta-\alpha V)=$ $O\left(\alpha^{q}\right)$. В двумерном случае (исследованном М. Ш. Бирманом совместно с А. А. Лаптевым, 1996) обнаружился интересный эффект - был найден класс потенциалов, для которых асимптотика $N_{-}(-\Delta-\alpha V)$ имеет вейлевский порядок $O(\lambda)$, а асимптотический коэффициент есть сумма вейлевского и "порогового" членов.

Аналогичные эффекты были изучены и для дискретного спектра оператора Дирака и периодического оператора Шрёдингера, возникающего во внутренних спектральных лакунах при возмущении убывающим потенциалом.

В последние 10-12 лет своей жизни М.Ш. Бирман основное внимание уделял спектральной теории периодических дифференциальных операторов. Совместно с Т. А. Суслиной он исследовал проблему абсолютной непрерывности спектра периодических операторов математической физики.

Спектр периодического эллиптического оператора состоит из замкнутых отрезков вещественной оси (спектральных зон). Если нет зон, вырождающихся в точку, то спектр оператора оказывается чисто абсолютно непрерывным. Впервые отсутствие вырожденных зон для периодического оператора Шрёдингера в $L_{2}\left(\mathbb{R}^{d}\right)$ было доказано Л. Томасом в 1973 г. Попытки обобщить результат Томаса на случай периодического 
магнитного оператора Шрёдингера терпели провал. После почти 25-летнего застоя в работе М. Ш. Бирмана и Т. А. Суслиной (1997) была установлена абсолютная непрерывность спектра магнитного периодического оператора Шрёдингера в размерности $d=2$. Эта работа была прорывом в данной области.

Последний цикл работ М. Ш. Бирмана (совместный с Т. А. Суслиной, 2000-2009) состоит в разработке нового теоретико-операторного подхода к теории усреднений (гомогенизации) периодических дифференциальных операторов.

Пусть $A=-\operatorname{div} g(\mathbf{x}) \nabla$ - эллиптический оператор в $L_{2}\left(\mathbb{R}^{d}\right)$ с периодической матрицей коэффициентов $g(\mathbf{x})$. Изучается оператор $A_{\varepsilon}=-\operatorname{div} g(\mathbf{x} / \varepsilon) \nabla, \varepsilon>0$, коэффициенты которого быстро осциллируют при малом $\varepsilon$. Типичная задача гомогенизации состоит в изучении поведения решения $u_{\varepsilon}$ уравнения $A_{\varepsilon} u_{\varepsilon}+u_{\varepsilon}=F$. Было известно, что при $\varepsilon \rightarrow 0$ решение $u_{\varepsilon}$ сходится (в некотором смысле) к решению $u_{0}$ "усредненного" уравнения $A^{0} u_{0}+u_{0}=F$. Здесь $A^{0}=-\operatorname{div} g^{0} \nabla$ - эффективный оператор, а $g^{0}-$ постоянная положительная эффективная матрица.

С помощью нового подхода впервые удалось доказать, что резольвента $\left(A_{\varepsilon}+I\right)^{-1}$ сходится к резольвенте $\left(A^{0}+I\right)^{-1}$ по операторной норме в $L_{2}$. Для нормы разности резольвент была получена оценка порядка $\varepsilon$. Основная идея состоит в том, что процесс усреднения можно изучать как спектральный пороговый эффект на краю спектра оператора $A$.

Подобные "операторные оценки погрешности", а также более точные аппроксимации резольвенты были получены для широкого класса матричных дифференциальных операторов. На основе разработанного метода изучалась гомогенизация в параболических задачах, для стационарной системы Максвелла и, наконец, для нестационарных уравнений типа Шрёдингера и гиперболического типа.

Несмотря на плохое состояние здоровья, Михаил Шлемович сохранял творческую активность до последних дней своей жизни. Он жил в науке и в профессии до конца. Память о Михаиле Шлемовиче Бирмане навсегда сохранится в сердцах тех, кому посчастливилось знать этого замечательного ученого и человека.

В. М. Бабич, В.С. Буслаев, А.М. Вершик, С.Г. Гиндикин, С.В. Кисляков, А.А. Лаптев, В.А. Марченко, Н.К. Никольский, Л. А. Пастур, Б. А. Пламеневский, М. З. Соломяк, Т. А. Суслина, Н. Н. Уральцева, Л. Д. Фаддеев, В.П. Хавин, Д. Р. Яфаев 